\title{
Are 2D:4D finger-length ratios an indicator of androgenetic alopecia in males?*
}

\author{
Özlem Bilgic ${ }^{1}$ \\ Dilek Eryılmaz ${ }^{1}$
}

\author{
Hilmi Cevdet Altınyazar ${ }^{1}$ \\ Zehra Ayça Tuğrul ${ }^{1}$
}

DOI: http:/ /dx.doi.org/10.1590/abd1806-4841.20164622

\begin{abstract}
BACKGROUND: Although the pathogenesis of androgenetic alopecia is not completely understood, the roles of genetic susceptibility and androgens are well-known. A lower ratio of the second digit (index finger $=2 \mathrm{D}$ ) to the fourth digit (ring finger $=4 \mathrm{D}$ ) length has been hypothesized to reflect prenatal androgen exposure and/or higher sensitivity to androgens.

OвJеCтIVEs: To determine the relationship between the second to fourth digit length ratio and androgenetic alopecia. Methods: Finger length measurements were made by a digital vernier calliper. Androgenetic alopecia severity was assessed using the Hamilton-Norwood scale. Subjects with an androgenetic alopecia score of grade III or more were included in the study.

RESULTS: A total of 189 males with androgenetic alopecia and 171 healthy controls were enrolled in the study. The age range of participants was 19-65 years. The 2D:4D ratios in patients with androgenetic alopecia were significantly lower than those of healthy controls for the right hand; however, no significant difference was found for the left hand. Average 2D:4D ratios in androgenetic alopecia patients were also lower than in controls. No significant relationship was observed between androgenetic alopecia severity and 2D:4D ratios.

CONCLUSION: Our data support the anatomical evidence of in utero androgen exposure and/or an individual's sensitivity to androgens in patients with androgenetic alopecia. Furthermore, the right hand 2D:4D ratio might be an indicator of androgenetic alopecia development.
\end{abstract}

Keywords: Alopecia; Androgens; Fingers; Hyperandrogenism; Receptors, androgen

\section{INTRODUCTION}

Androgenetic alopecia (AGA) is the most common form of hair loss and may begin at any age after puberty. Although the exact prevalence is unknown and varies among races, almost $50 \%$ of men are affected by their fourth decade of life, and prevalence rates reach approximately $80 \%$ in Caucasian men by the seventh decade of life. ${ }^{1-3}$ The pathophysiology of AGA is not completely understood but circulating androgens, increased $5 \alpha$-reductase type II activity, androgen receptors (ARs) and their coactivators, as well as some paracrine mediators (e.g., TGF- $\beta 1$ ) are blamed for the development of AGA in genetically susceptible men. ${ }^{1-6}$

The ratio of the second digit (index finger $=2 \mathrm{D}$ ) to the fourth digit (ring finger $=4 \mathrm{D}$ ) length has been hypothesized to reflect prenatal androgen exposure and the individual's sensitivity to androgens. ${ }^{78} \mathrm{Un}$ - like in females, the $4 \mathrm{D}$ in males is usually longer than the 2D (lower 2D:4D). This gender difference in digit length ratios has been linked with the in utero balance of androgen to oestrogen. It has also been suggested that the 2D:4D ratio is correlated negatively with prenatal testosterone levels. ${ }^{7-9}$ To support this hypothesis, direct relationships have been shown between foetal testosterone and 2D:4D by analysing the amniotic fluid. ${ }^{10}$ Moreover, experimentally manipulated hormone levels in pregnant rats have revealed a relationship between increased levels of maternal testosterone and lower 2D:4D ratios in offspring. ${ }^{11}$ In addition to higher androgen levels, there is evidence showing a relationship between ARs and 2D:4D ratios. ${ }^{12-14}$ Higher (female-type) digit ratios are found in chromosomally male individuals with androgen insensitivity syn-

\footnotetext{
Received on 07.04.2015.

Approved by the Advisory Board and accepted for publication on 21.06.2015.

Work performed at the School of Medicine, Selcuk University, Department of Dermatology - Konya, Turkey.

Financial Support: None.

Conflict of Interest: None.

Selcuk University - Konya, Turkey.

C2016 by Anais Brasileiros de Dermatologia
} 
drome. ${ }^{14}$ Furthermore, the AR gene polymorphism has been correlated with 2D:4D. This presents as a low cytosine-adenine-guanine (CAG) number with a low 2D:4D, indicating increased androgen responsiveness with more sensitive androgen receptors. ${ }^{12-14}$

Hair follicles in the human foetus first occur at approximately 9 weeks of gestation, with most developing by week 16, coinciding closely with the peak of prenatal androgens and the determination of digit ratios. ${ }^{14,15}$ To date, many studies have evaluated the putative impact of prenatal androgens on physical, psychological and medical characteristics by using the 2D:4D ratio. ${ }^{8}$ However, the relationship between AGA and the 2D:4D ratio has not yet been investigated. This study sought to determine the relationship between the digit ratio and AGA in males to provide evidence that the 2D:4D ratios are an indicator of AGA.

\section{METHODS}

This study was approved by the Institutional Review Board and informed consent was given by all participants. The study group comprised male patients with AGA and healthy, male controls who attended the dermatology outpatient clinic. All admissions for AGA, excluding those with female pattern hair loss, were considered eligible for the study. The control group consisted of selected patients who attended a dermatologic consultation on a matter other than AGA.

Participants with deformities on the hands or afflicted/injured fingers were excluded from this study. It also excluded individuals who had taken drugs (e.g. glucocorticoid medication, hormone replacement therapy) that could have affected hair growth in the preceding three months, or who had endured systemic diseases related to diffuse hair loss (e.g. thyroid, pituitary, adrenal or collagen diseases). Furthermore, the study excluded patients who were using any topical or systemic medication for androgenetic alopecia, as well as those who had undergone a hair transplantation.

AGA severity was assessed using the Hamilton-Norwood (HN) scale and subjects with AGA grade III or more were included in the study. ${ }^{2-4}$ Finger-length was measured via a digital vernier calliper with a resolution of $0.01 \mathrm{~mm}$. Measurements of the ring and index fingers were made directly on the palmar surface of both hands, from the basal crease (metacarpophalangeal crease) to the tip. If more than one fold was detected at the base of the finger, the most proximal fold was used. To obtain the 2D:4D ratio, the length of the index finger was divided by the length of the ring finger.

We used the statistical software SPSS 17.0 (SPSS Inc., Chicago, IL, USA) to analyse data. When normality of variable distribution was acceptable, the Stu- dent's t-test or the Mann-Whitney U-test were used to analyse differences between groups. Cohen's effect size was calculated to quantify the extent of the difference in 2D:4D between individuals with AGA and controls. Standardized Cohen's $d ; 0.2,0.5$, and 0.8 were used to indicate the mild, moderate and large effect sizes, respectively. Spearman correlation coefficients examined relationships between digit ratios and AGA severity. A value of $P<0.05$ (two-tailed test) was considered significant.

\section{RESULTS}

A total of 189 males with AGA and 171 healthy controls participated in the study. The median age (range: 19-65 years) did not differ significantly between patient (median $=36$, interquartile range $=20.5$ ) and control (median $=35$, interquartile range $=13$ ) groups $(\mathrm{z}=-1.11, p=0.266)$. The distribution of AGA severity in subjects is shown in table 1 .

The 2D:4D ratios of the right and left hands (taken separately) and the average 2D:4D ratio for AGA patients were compared to those of healthy controls. In AGA patients, the 2D:4D ratios were significantly lower than in healthy controls for the right hand; however, we found no significant difference for the left hand. Average 2D:4D ratios in AGA subjects were also lower than for the controls. Cohen's d yielded mild to moderate and mild effect sizes for the right and average digit ratios, respectively. Table 2 summarizes the findings of the 2D:4D ratios in AGA and control groups.

Correlations between the severity of AGA and 2D:4D ratios were evaluated in the AGA group. No significant relationships occurred between AGA severity and right $\left(r_{s}=-0.10, p=0.179\right)$, left $\left(r_{s}=0.60, p=\right.$ $0.445)$ or average $\left(r_{s}=-0.03, p=0.707\right) 2 \mathrm{D}: 4 \mathrm{D}$ ratios.

TABLE 1: Distribution of androgenetic alopecia severity for the patients' group according to Hamilton Norwood classification

\begin{tabular}{ll}
\hline AGA severity & $\begin{array}{l}\text { Patients } \\
(\mathbf{n}=\mathbf{1 8 9})\end{array}$ \\
\hline Norwood 3 (n/\%) & $49 / 25.9$ \\
Norwood 3a (n/\%) & $9 / 4.8$ \\
Norwood 3 vertex (n/\%) & $42 / 22.2$ \\
Norwood 4 (n/\%) & $27 / 14.3$ \\
Norwood 4a (n/\%) & $3 / 1.6$ \\
Norwood 5 (n/\%) & $23 / 12.2$ \\
Norwood 5a (n/\%) & $12 / 6.3$ \\
Norwood 6 (n/\%) & $18 / 9.5$ \\
Norwood 7 (n/\%) & $6 / 3.2$ \\
\hline
\end{tabular}

AGA: Androgenetic alopecia 
TABLE 2: Comparison of the 2D:4D ratios for males with androgenetic alopecia and control subjects

\begin{tabular}{|c|c|c|c|c|c|c|c|c|c|}
\hline & \multicolumn{3}{|c|}{ Patients (n=189) } & \multicolumn{3}{|c|}{ Controls $(n=171)$} & \multicolumn{3}{|c|}{ Analysis } \\
\hline & Mean & SD & Range & Mean & SD & Range & $t$ & $\mathbf{p}$ & Cohen's d \\
\hline Right 2D:4D & 0.968 & 0.032 & $0.89-1.07$ & 0.980 & 0.030 & $0.88-1.10$ & -3.539 & $<0.001$ & -0.386 \\
\hline Left 2D:4D & 0.971 & 0.030 & $0.90-1.04$ & 0.976 & 0.032 & $0.89-1.06$ & -1.641 & 0.100 & -0.161 \\
\hline Average 2D:4D & 0.970 & 0.029 & $0.89-1.04$ & 0.978 & 0.029 & $0.88-1.06$ & -2.778 & 0.005 & -0.275 \\
\hline
\end{tabular}

\section{DISCUSSION}

To our knowledge, this is the first study that has investigated the relationship between AGA and 2D:4D ratios. We found that a lower $2 \mathrm{D}: 4 \mathrm{D}$ ratio in the right hand is associated with AGA in males with a mild to moderate effect size. Since previous reports suggested a relationship between low 2D:4D and prenatal androgen exposure, this study supports the idea that prenatal androgen has a role in the pathogenesis of AGA.

Prenatal androgen levels peak at the end of the first trimester and cause some organizing effects on a number of organ systems with a lifelong impact. ${ }^{8}$ For example, foetal androgen excess in women stimulates masculine behaviours besides urogenital virilization. ${ }^{16}$ In female babies with congenital adrenal hyperplasia, even if treatments start immediately after birth, the risk of gender confusion increases in the future because of the brain virilization caused by elevated prenatal androgen levels. ${ }^{17}$ Furthermore, in utero exposure to excessive androgens leads to enlarged and polyfollicular ovaries during adulthood in female rhesus monkeys and sheep, similarly to polycystic ovary syndrome in women. ${ }^{18} \mathrm{~A}$ study on the long-lasting impact of prenatal androgens on pilosebaceous units yielded similar results by observing increased sebum secretion in adulthood in rats that had been exposed to androgens in the first week of foetal life. ${ }^{19}$ Our finding of a lower 2D:4D ratio in males with AGA is consistent with previous reports on the impact of foetal androgens on organ systems in later life. However, nothing more than a mild to moderate association was found between digit ratio and AGA, suggesting that prenatal androgen exposure only partly explains the etiopathogenesis of AGA.

In hair follicles, testosterone is converted to its fivefold potent metabolite-dihydrotestosterone by the $5 \alpha$-reductase type II enzyme, and then binds to ARs. ${ }^{1}$ This binding changes the hair cycle dynamic by shortening the growth (anagen) duration and elongating the rest (telogen) duration, leading to hair miniaturization and eventually baldness. ${ }^{5}$ It is thought that ARs determine the sensitivity of follicular cells to androgens by specific polymorphisms in the AR gene. Moreover, some AR gene polymorphisms (e.g. shorter CAG re- peats in ARs) have been more prevalently identified in AGA patients. ${ }^{3,20}$ Moreover, AGA does not feature in men with a genetic absence of $5 \alpha$-reductase type II or functional androgen receptors (e.g. androgen insensitivity syndrome) $)^{4}$. Interestingly, as noted above, both shorter CAG repeats in ARs and androgen insensitivity syndrome have been linked to lower and higher 2D:4D ratios, respectively. Thus, our finding regarding the association between lower digit ratios and AGA may indicate increased androgen responsiveness with more sensitive androgen receptors.

Given our a priori hypothesis, we expected that lower 2D:4D ratios would show a negative correlation with AGA severity, indicating more severe AGA. However, we found no significant correlation between 2D:4D and AGA severity. This suggests that although both lower 2D:4D ratios and AGA may share a common etiological mechanism, other factors unrelated to 2D:4D could account for AGA severity.

Regarding the limitations of this study, we cannot rule out the possibility that our sample is biased since the experimenters who measured the digit ratios were not blind to the subjects' AGA status. If there was a bias, we could have expected to find similar results for both hands, yet we failed to find any relationship between digit ratios and AGA in the left hand. Additionally, we would expect to find a negative relationship between AGA severity and the digit ratio. Contrary to our hypothesis, we found no relationship between these two variables. This finding also supports the notion that there was no bias in our measurements.

\section{CONCLUSION}

Our data support the anatomical evidence of in utero androgen exposure and/or an individual's sensitivity to androgens in AGA patients, and show that the right hand 2D:4D ratio may be a predictor of AGA development. Future studies with larger sample sizes will increase our knowledge of prenatal androgen exposure and its potential consequences for AGA patients. $\square$ 


\section{REFERENCES}

1. Rhodes T, Girman CJ, Savin RC, Kaufman KD, Guo S, Lilly FR, et al. Prevalence of male pattern hair loss in 18-49 year old men. Dermatol Surg. 1998;24:1330-2.

2. Blume-Peytavi U, Blumeyer A, Tosti A, Finner A, Marmol V, Trakatelli M, et al. European Consensus Group. S1 guideline for diagnostic evaluation in androgenetic alopecia in men, women and adolescents. Br J Dermatol. 2011;164:5-15.

3. Kucerova R, Bienova M, Kral M, Bouchal J, Trtkova KS, Burdova A, et al. Androgenetic alopecia and polymorphism of the androgen receptor gene (SNP rs6152) in patients with benign prostate hyperplasia or prostate cancer. J Eur Acad Dermatol Venereol. 2015;29:91-6.

4. Ellis JA, Sinclair R, Harrap SB. Androgenetic alopecia: pathogenesis and potential for therapy. Expert Rev Mol Med. 2002;4:1-11.

5. Otberg N, Finner AM, Shapiro J. Androgenetic alopecia. Endocrinol Metab Clin North Am. 2007;36:379-98.

6. Inui S, Itami S. Molecular basis of androgenetic alopecia: From androgen to paracrine mediators through dermal papilla. J Dermatol Sci. 2011;61:1-6.

7. Hönekopp J, Watson S. Meta-analysis of digit ratio 2D:4D shows greater sex difference in the right hand. Am J Hum Biol. 2010;22:619-30.

8. Manning JT. Resolving the role of prenatal sex steroids in the development of digit ratio. Proc Natl Acad Sci U S A. 2011;108:16143-4.

9. Zheng Z, Cohn MJ. Developmental basis of sexually dimorphic digit ratios. Proc Natl Acad Sci U S A. 2011;108:16289-94.

10. Lutchmaya S, Baron-Cohen S, Raggatt P, Knickmeyer R, Manning JT. 2nd to 4th digit ratios, fetal testosterone and estradiol. Early Hum Dev. 2004;77:23-8.

11. Talarovicová A, Krsková L, Blazeková J. Testosterone enhancement during pregnancy influences the $2 \mathrm{D}: 4 \mathrm{D}$ ratio and open field motor activity of rat siblings in adulthood. Horm Behav. 2009;55:235-9.

12. Manning JT, Bundred PE, Newton DJ, Flanagan BF. The second to fourth digit ratio and variation in the androgen receptor gene. Evol Hum Behav. 2003;24:399-405.

13. Manning JT, Bundred PE, Flanagan BF. The ratio of 2nd to 4th digit length: a proxy for transactivation activity of the androgen receptor gene? Med Hypotheses. 2002;59:334-6.

14. Byne W. Developmental endocrine influences on gender identity: implications for management of disorders of sex development. Mt Sinai J Med. 2006;73:950-9.

15. Paus R, Stefan Peker S, Sundberg JP. Biology of Hair and Nails. In: Bolognia JL, Jorizzo JL, Rapini RP editors. Dermatology 2nd ed. Spain: Mosby Elsevier; 2008.p. 965-86.
16. Abbott DH, Padmanabhan V, Dumesic DA. Contributions of androgen and estrogen to fetal programming of ovarian dysfunction. Reprod Biol Endocrinol. 2006;4:17.

17. Berenbaum SA. Effects of early androgens on sex-typed activities and interests in adolescents with congenital adrenal hyperplasia. Horm Behav. 1999 Feb;35(1):102-10.

18. Abbott DH, Barnett DK, Bruns CM, Dumesic DA. Androgen excess fetal programming of female reproduction: a developmental aetiology for polycystic ovary syndrome? Hum Reprod Update. 2005;11:357-74.

19. Zouboulis CC, Fimmel S, Ortmann J, Turnbull JR, Boschnakow A. Sebaceous Glands. In: Hoath SB, Maibach HI, editors. Neonatal Skin: Structure and Function. 2nd ed. New York: Marcel Dekker; 2003.p. 59-87.

20. Zhuo FL, Xu W, Wang L, Wu Y, Xu ZL, Zhao JY. Androgen receptor gene polymorphisms and risk for androgenetic alopecia: a meta-analysis. Clin Exp Dermatol. 2012;37:104-11.

\author{
MAILING ADDRESS: \\ Özlem Bilgiç \\ Selçuk Üniversitesi Tıp Fakültesi Hastanesi \\ Deri ve Zührevi Hastalıklar Anabilim Dalı \\ Alaeddin Keykubad Kampüsü \\ 42075 - Selçuklu \\ Konya - TURKEY \\ E-mail: bilgicozlem@yahoo.com
}

How to cite this article: Bilgic O, Altinyazar HC, Eryilmaz D, Tugrul ZA. Are the 2D:4D finger length ratios an indicator of androgenetic alopecia in males? An Bras Dermatol. 2016;91(2):156-9. 